\title{
The effect of surface roughness on the convective instability of the BEK family of boundary-layer flows
}

\author{
B. Alveroglu ${ }^{1}$, A. Segalini ${ }^{2}$, and S.J. Garrett ${ }^{3}$ \\ ${ }^{1}$ Department of Mathematics, University of Leicester, Leicester, LE1 7RH, UK \\ ${ }^{2}$ Linné FLOW Centre, KTH Mechanics, SE-100 44, Stockholm, Sweden \\ ${ }^{3}$ Department of Engineering, University of Leicester, Leicester, LE1 7RH, UK
}

October 15, 2015

\begin{abstract}
A Chebyshev polynomial discretisation method is used to investigate the effect of both anisotropic (radially and azimuthally) and isotropic surface roughness on the convective instability of the BEK family of rotating boundary-layer flows. The mean-flow profiles for the velocity components are obtained by modelling surface roughness with a partialslip approach. A linear stability analysis is then performed to investigate the effect of roughness on the convective instability characteristics of the inviscid Type I (cross-flow) instability and the viscous Type II instability. It is revealed that all roughness types lead to a stabilisation of the Type I mode in all flows within the BEK family, with the exception of azimuthally-anisotropic roughness (radial grooves) within the Bödewadt layer which causes a mildly destabilising effect. In the case of the Type II mode, the results reveal the destabilising effect of radially-anisotropic roughness (concentric grooves) on all the boundary layers, whereas both azimuthally-anisotropic and isotropic roughness have a stabilising effect on the mode for Ekman and von Kármán layers. Complementary results are also presented by considering the effects of roughness on the growth rates of each instability mode within the Ekman layer.
\end{abstract}




\section{Introduction}

2 Viscous shear forces acting on the surface of a rigid body cause skin-friction drag when the 3 body moves through a fluid. In most technological applications, this drag increases as the flow

4 transitions from laminar to turbulent and finding a way to reduce drag has been a priority

5 for many years. It was widely believed that smooth surfaces are likely to generate less skinfriction drag than non-smooth (rough) surfaces, however, more recent studies have shown this is not necessarily always the case ${ }^{1-3}$. Indeed it has been revealed that properly designed surface roughness could be used to develop new drag-reduction techniques ${ }^{2-4}$.

Since lower drag forces result from laminar boundary layers, a promising strategy towards drag reduction is to delay the transition of a laminar boundary-layer flow via surface roughness. This approach was demonstrated very recently by Cooper et al. ${ }^{4}$ for the von Kármán ${ }^{5}$ boundary-layer flow established over a disk rotating in a still fluid. In this current paper we extend those results with the introduction of an additional rotating disk in the far-field above. ${ }_{4}$ This results in the "BEK" class of flows with the differential rotation rate included as a key 15 parameter. This broad class of boundary-layer flows, that includes the Bödewadt ${ }^{6}$, Ekman ${ }^{7}$ and von Kármán ${ }^{5}$ flows, occurs in turbo-machinery and rotor-stator devices, such as mixers, and their stability characteristics therefore have practical importance. Here we are motivated by turbo-machinery applications where laminar flow should be maintained as long as possible and lower-disk surface roughness is a potential stabilising mechanism.

The similarities in the stability characteristics of all flows within the BEK class are well known (see, for example, the theoretical and experimental studies discussed in papers ${ }^{8-13}$ for the Bödewadt layer; papers ${ }^{14 ; 15}$ for the Ekman layer; and papers ${ }^{16-19}$ for the von Kármán flow). In all these flows an inflectional cross-flow velocity component causes a characteristic flow instability that is inviscid in nature: this is commonly referred to as the Type I instability and tends to be dominant over smooth disks. In addition there exists a further Type II mode that is viscous in nature and caused by streamwise curvature and Coriolis effects. The literature shows that spiral patterns of co-rotating vortices associated with the convective instabilities, and rotating with the lower disk, have been observed in regions outside the turbulent regime for all flows of the BEK family. Although modes travelling relative to the lower disk surface are known to exist and, in some cases, are theoretically dominant, in practice the disturbances 
are excited by unavoidable roughnesses on the smooth-disk surface. Since these roughnesses are fixed in time in the rotating frame, stationary disturbances are consistently excited and reinforced such that they are evident in flow-visualizations and quantitative experiments. We also note that travelling Type I modes contribute to the well-known local absolute instability in the entire class of BEK flows ${ }^{16 ; 28}$.

Until recently, the literature has focussed on understanding the characteristics of laminarturbulent transition over smooth disks. Interest in rough disks is increasing and some valuable contributions to understanding the effects of surface roughness on the transition process have already been made. Work in this area began with the use of small numbers of roughness elements aligned in particular patterns and targeted to excite particular disturbance modes within the boundary layer ${ }^{20-23}$. However, studies involving more general distributed surface roughness (as is our interest here) can be considered as starting with the Watanabe et al. ${ }^{24}$ study of the related boundary-layer flow over the rotating cone. Their experimental results revealed that modest levels of surface roughness can decrease the number of vortices from 32 (for a smooth surface) to 26 (for a rough surface), thereby hinting at the potentially stabilising nature of the roughness. The connection between the rotating cone boundary layer and the rotating disk is well known ${ }^{25}$ and the Watanabe et al. study prompted the very recent theoretical work of Cooper et al. ${ }^{4}$ who studied the effects of surface roughness on the von Kármán flow.

Two theoretical models for the steady boundary-layer flow over rough disks have been developed in the literature by Miklavčič \& Wang ${ }^{26}$ and Yoon et al. ${ }^{27}$, henceforth referred to as the MW and YHP models, respectively. Both models demonstrate how surface roughness could lead to modifications to the classic similarity solution for the flow over a smooth disk due to von Kármán ${ }^{5}$. Both models are however fundamentally different. The YHP approach models roughness by imposing a particular surface distribution as a function of radial position only and assumes a rotational symmetry, a particular case of anisotropic roughness. Alternatively, rather than imposing a particular mathematical form of the surface roughness, the MW approach models roughness empirically by replacing the usual no-slip boundary conditions with partialslip conditions at the disk surface. The MW approach can therefore model independent levels of roughness in the radial and azimuthal directions by separately modifying the boundary conditions in these directions. This formulation thereby permits isotropic roughness (uniform in all directions) and also all variations of anisotropic roughness. The flexibility of the MW 
approach is a significant advantage and it will be used throughout this paper.

Cooper et al.'s study ${ }^{4}$ used the full flexibility of the MW model to consider the effects of isotropic roughness and two forms of anisotropic roughness: concentric grooves (radial anisotropic) and radial grooves (azimuthal anisotropic), on the von Kármán flow. Their results reveal that both anisotropic and isotropic roughness lead to a stabilisation of the Type I instability mode (in terms of increased critical Reynolds number). In addition, in the case of radial anisotropic roughness, a significant destabilisation of the Type II mode is found. In the particular case of the von Kármán flow at least, the emergence of the Type II mode with increasing roughness should therefore be understood as halting the beneficial roughness effects on the Type I mode.

This current paper proceeds as follows. The mean-flow equations and calculations of the steady boundary-layer flows of the BEK system over rough rotating disks are presented in Section 2. We then proceed to perform linear stability analyses on these flows in Section 3. We present neutral curves along with detailed predictions of measurable quantities, such as number of co-rotating vortices, critical Reynolds numbers and the vortex-orientation angle. Our conclusions are drawn in Section 4.

\section{The steady mean flows}

The boundary-layer flows of the BEK system are distinguished by a differential rotation rate between the lower disk and the fluid above (generated by the upper rotating disk) as the system spins within an otherwise still, incompressible fluid. Both the lower disk and upper fluid are assumed to be infinite and they rotate around a common axis with angular velocities $\Omega_{D}^{*}$ and $\Omega_{F}^{*}$, respectively. Whilst there is a continuum of cases in which the disk and fluid rotate independently with different angular velocities, three particular cases correspond to standard flow configurations: the Bödewadt flow has $\Omega_{D}^{*}=0$ and $\Omega_{F}^{*} \neq 0$; the Ekman flow has $\Omega_{D}^{*}=\Omega_{F}^{*}$; and the von Kármán flow has $\Omega_{D}^{*} \neq 0$ and $\Omega_{F}^{*}=0$. Although the formulation of the model does not preclude a counter-rotating disk and upper fluid, it is assumed throughout this paper that both $\Omega_{D}^{*}, \Omega_{F}^{*}>0$, that is, the disk and fluid rotate in the same direction.

The steady-flow formulation is entirely consistent with the related papers of Lingwood ${ }^{16}$ and Lingwood \& Garrett ${ }^{28}$ and full details of the scalings and derivations of the governing equations can be found there. Here it is sufficient to state that we work in a cylindrical polar 
coordinate system that rotates with the lower disk. The steady mean-flow profiles can then be

93 shown to be determined by the ODE system

$$
\begin{array}{r}
R o\left\{U^{2}+U^{\prime} W-\left(V^{2}-1\right)\right\}-C o(V-1)-U^{\prime \prime}=0, \\
R o\left(2 U V+V^{\prime} W\right)+C o U-V^{\prime \prime}=0, \\
R o\left(W W^{\prime}+P^{\prime}\right)-W^{\prime \prime}=0, \\
2 U+W^{\prime}=0,
\end{array}
$$

where all the derivatives (denoted by a prime) are with respect to $z$, the scaled distance through the boundary layer in the wall-normal (i.e. axial) direction. The variables $U, V$ and $W$ are the components in the radial, azimuthal and axial directions, respectively, and $P$ is the pressure. The parameters $R o$ and $C o$ are the constant Rossby and Coriolis numbers such that $C o=$ $2-R o-R o^{2}$ and

$\begin{array}{lll}\text { Bödewadt flow: } & \text { Ro }=1 & C o=0 \\ \text { Ekman flow: } & R o=0 & C o=2 \\ \text { von Kármán flow: } & R o=-1 & C o=2\end{array}$

${ }_{94}$ We proceed to use the MW model ${ }^{26}$ to determine the boundary conditions. As discussed 95 in Section 1, this approach modifies the no-slip condition at the disk surface, $z=0$, whereas the far-field boundary conditions at the upper edge of the boundary layer are identical to the smooth-disk formulation. In particular, we find that the ODE system (1) is subject to the conditions

$$
\begin{array}{ll}
U=\lambda U^{\prime}, & V=\eta V^{\prime} \quad \text { and } \quad W=0 \quad \text { at } z=0, \\
U=0, & V=1 \quad \text { as } z \rightarrow \infty .
\end{array}
$$

99 Here the differentiation is again with respect to $z$ and the two parameters $\eta$ and $\lambda$ are empirical measures of roughness in the radial and azimuthal directions, respectively. The no-slip boundary conditions of a smooth disk are recovered when $\lambda=\eta=0$. The particular cases $\eta>0, \lambda=0$ (concentric grooves) and $\eta=0, \lambda>0$ (radial grooves) correspond to radially- and azimuthallyanisotropic roughness, respectively, while the case $\eta=\lambda>0$ corresponds to isotropic roughness.

For the Ekman layer, $R o=0$, we note a precise analytical solution of equations (1) subject 
to boundary conditions (2) at general $\eta$ and $\lambda$,

$$
\begin{aligned}
U & =e^{-z}(A \sin z-B \cos z) \\
V & =1+e^{-z}(B \sin z+A \cos z) \\
W & =e^{-z}[B(\sin z-\cos z)+A(\sin z+\cos z)]+B-A,
\end{aligned}
$$

where $A$ and $B$ are constants given by

$$
A=-\frac{1+\lambda}{(1+\lambda)(1+\eta)+\lambda \eta} \quad \text { and } \quad B=\frac{\lambda}{(1+\lambda)(1+\eta)+\lambda \eta}
$$

For $R o \neq 0$, no such analytical solution exists and a shooting method consistent with the literature $^{28}$ is incorporated to solve the governing equations between $\operatorname{Ro} \in[-1,1]$.

The computed flow profiles for the Ekman layer with lower disk surface roughness are presented in Figures 1 and 2. In the case of radially-anisotropic roughness $(\eta \neq 0, \lambda=0)$, Figure 1(a) shows that there is a slight modification of the boundary-layer thickness and a reduction in the radial jet as the roughness parameter $\eta$ is increased. The effect of non-zero $\eta$ on the azimuthal profile is an incremental increase of its value at the disk surface. This is shown in Figure 1(c) and is a direct implication of the partial-slip condition. A reduction in the magnitude of the fluid in the far-field is shown in Figure 1(e) as a result of increasing $\eta$. Furthermore, we note that the slight 'oscillatory' behaviour of each flow component is seen to be damped out as $\eta$ is increased. In contrast, in the case of azimuthally-anisotropic roughness $(\eta=0, \lambda \neq 0)$, there is an incremental increase in the value of the radial profile at the disk surface, an increase in the wall jet, and a slight thinning of the boundary layer with increased roughness parameter $\lambda$, as is evident in Figure 1(b). Figures 1(d) and 1(f) show that the azimuthal velocity is relatively unchanged but there is an increase in magnitude of the fluid in the far-field with increased $\lambda$. Figure 2 shows the effect of isotropic surface roughness $(\eta=\lambda \neq 0)$ on the Ekman layer. Our results clearly predict a reduction in the radial wall jet, a reduction in the magnitude of the fluid in the far-field and a general thinning effect.

The computed flow profiles for the Bödewadt layer, $R o=1$, with lower disk surface roughness are presented in Figures 3 and 4. The results for each particular type of roughness are qualitatively similar to those reported for the Ekman later. In particular, an increase in radiallyanisotropic roughness reduces both the wall jet and the magnitude of the fluid in the far-field, 


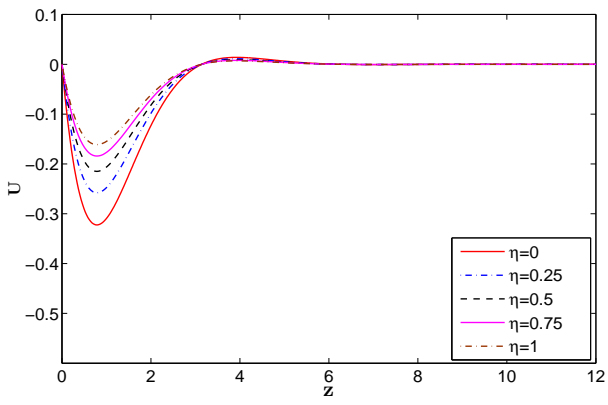

(a) $U$-profile

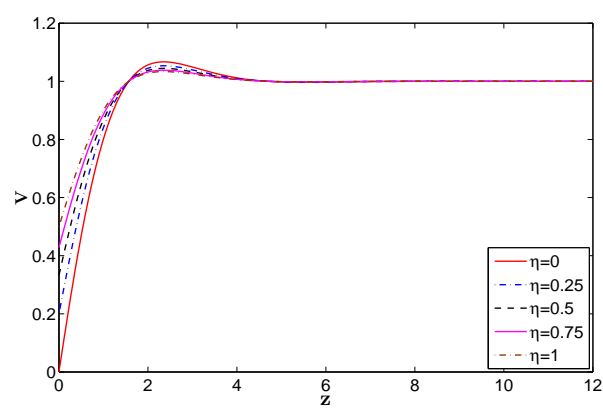

(c) $V$-profile

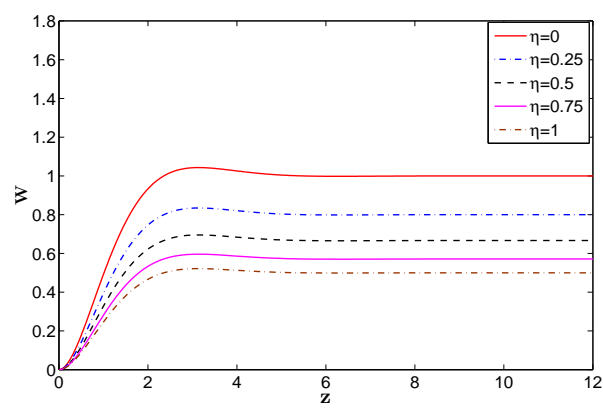

(e) $W$-profile

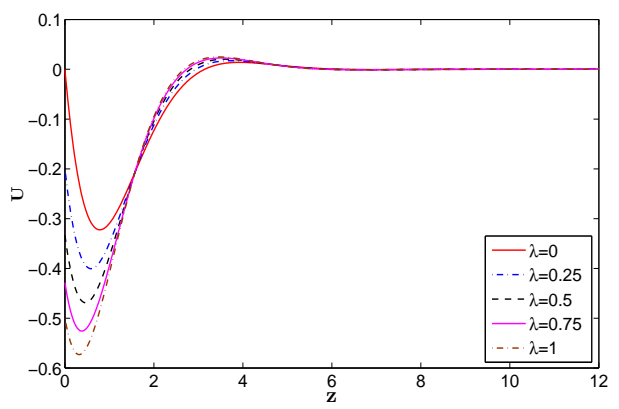

(b) $U$-profile

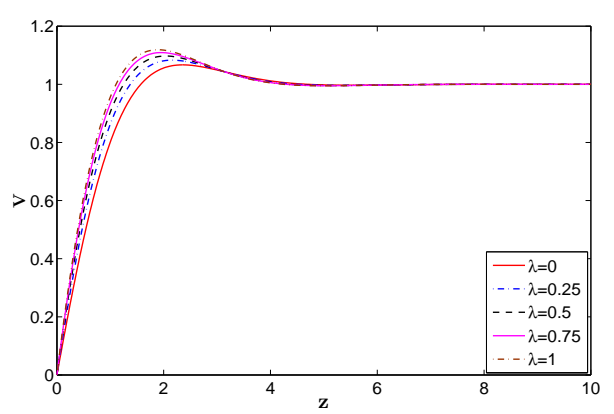

(d) $V$-profile

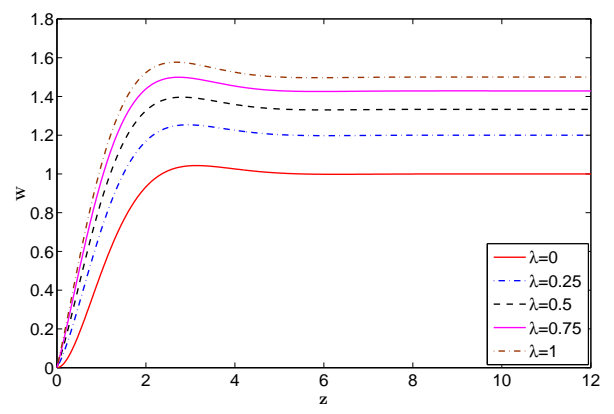

(f) $W$-profile

Figure 1: Mean-flow components of the Ekman flow $(R o=0)$ over rough disks: (a)-(c)-(e) radially-anisotropic roughness (concentric grooves, $\lambda=0$ ); (b)-(d)-(f) azimuthally-anisotropic roughness (radial grooves, $\eta=0$ ). 


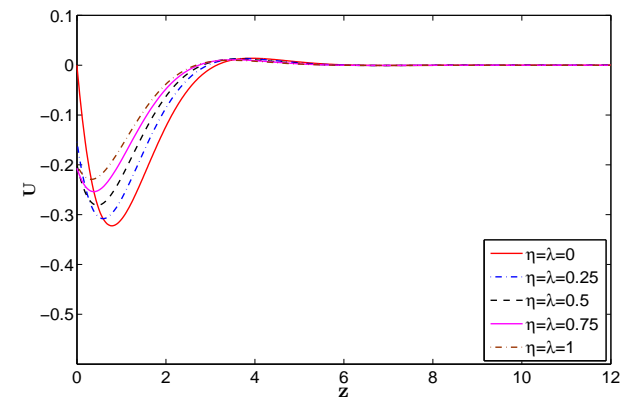

(a) $U$-profile

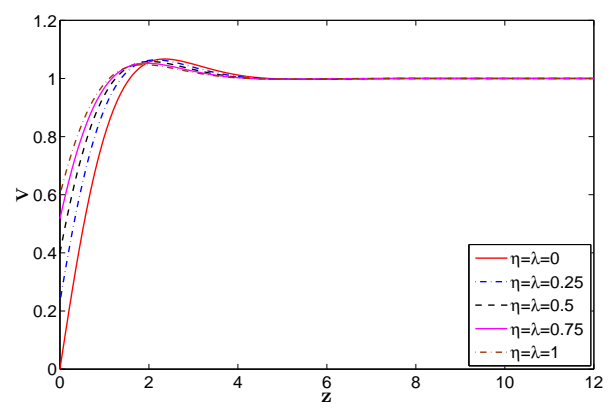

(b) $V$-profile

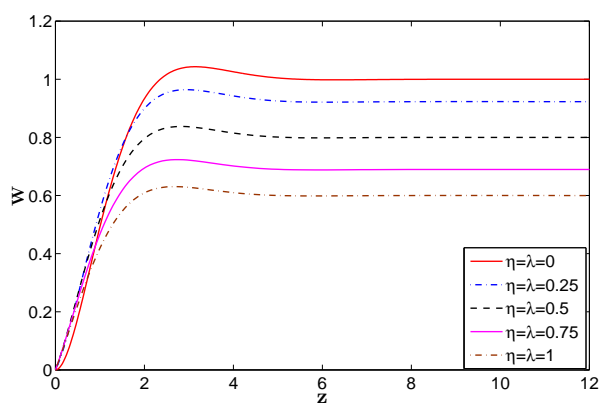

(c) $W$-profile

Figure 2: Mean-flow components of Ekman flow over isotropically rough disks. 


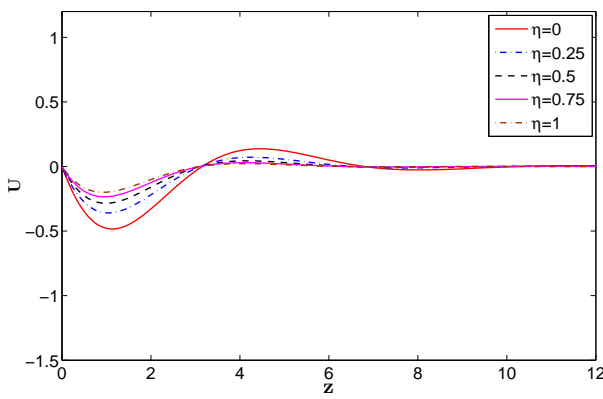

(a) $U$-profile

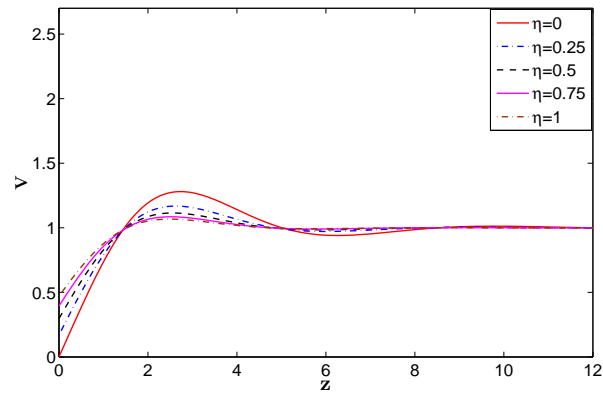

(c) $V$-profile

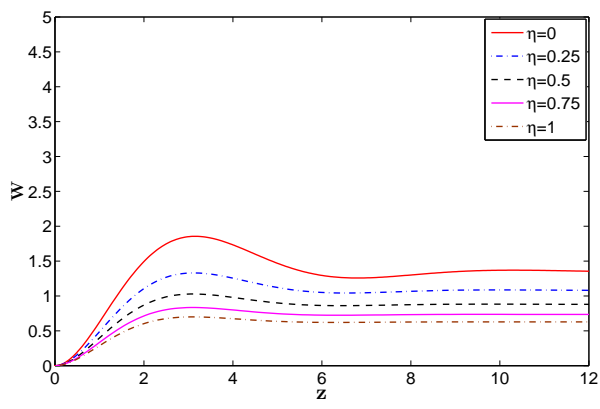

(e) $W$-profile

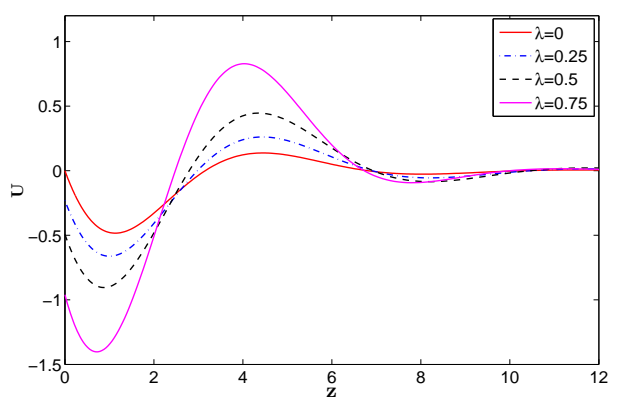

(b) $U$-profile

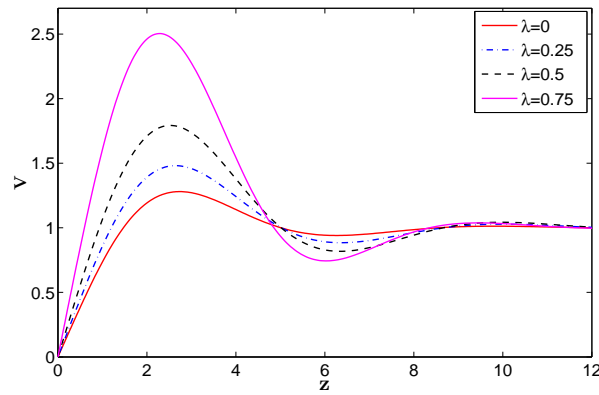

(d) $V$-profile

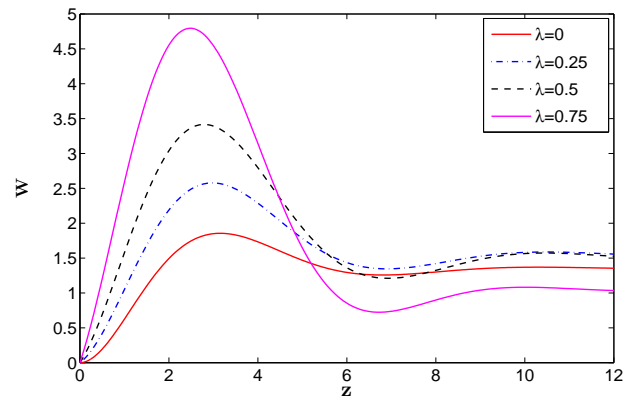

(f) $W$-profile

Figure 3: Mean flow components of Bödewadt flow $(R o=1)$ over rough disks: (a)-(c)-(e) radially-anisotropic roughness (concentric grooves, $\lambda=0$ ); (b)-(d)-(f) azimuthally-anisotropic roughness (radial grooves, $\eta=0$ ). 


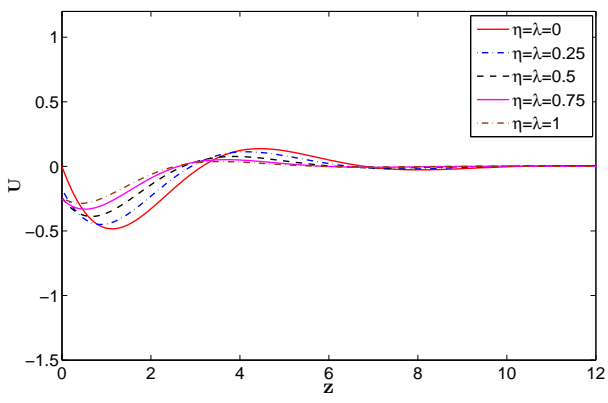

(a) $U$-profile

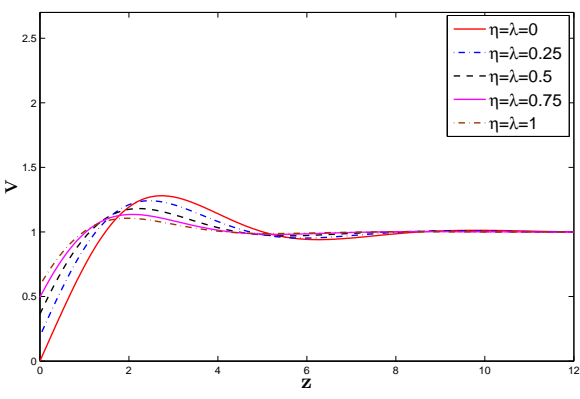

(b) $V$-profile

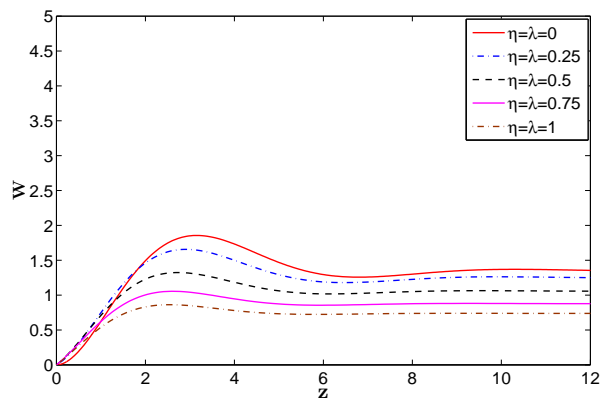

(c) $W$-profile

Figure 4: Mean-flow components of the Bödewadt flow over isotropically rough disks. 
whilst significantly damping the pronounced oscillatory nature. In contrast, an increase in the azimuthally-anisotropic roughness increases the wall jet and emphasises the oscillatory nature of the flow. The effect of increasing the level of isotropic surface roughness is also seen to reduce the wall jet, reduce the magnitude of the fluid in the far-field and thin the boundary layer. The oscillatory behaviour is again significantly damped.

The effects of each roughness type on the radial jet (which is seen to be the primary response to increasing surface roughness of each boundary layer within the BEK family) are consistent with those obtained by Cooper et al. ${ }^{4}$ for the von Kármán flow. That is, isotropic and radiallyanisotropic roughness acts to reduce the magnitude of the well jet, and azimuthally-anisotropic roughness acts to increase its magnitude. Furthermore, the effects of roughness on the oscillatory behaviour of the Ekman and Bödewadt flows are consistent with the responses seen to surface mass flux as reported by Lingwood \& Garrett ${ }^{28}$. In particular, Lingwood \& Garrett found suction to dampen the oscillatory behaviour and blowing to magnify it for all Ro. The effects of isotropic and radially-anisotropic roughness are therefore seen to be quantitatively consistent with surface suction and azimuthally-anisotropic roughness with surface blowing.

\section{Convective instability analysis}

\subsection{Formulation}

A local linear stability analysis is conducted on the steady, mean-flow profiles obtained in Section 2. As discussed by Cooper et al., the partial-slip formulation used here is such that the derivation of the perturbation equations is unchanged from the smooth-surface case. In this analysis of the BEK family the perturbation system is related to that described by Lingwood ${ }^{16}$ and we introduce a Reynolds number $R e=r_{a} R o$ where $r_{a}$ is the non-dimensional location of the analysis. For negative Rossby number the Reynolds number is also negative, although this is a consequence of the formulation used and results will be presented in terms of positive $R e$ for all Ro. We see that the Reynolds number can be interpreted as the non-dimensional location of the local analysis, and increasing $R e$ in magnitude corresponds to moving radially outwards from the axis of rotation (irrespective of the sign of the Rossby number). 


$$
(\hat{u}, \hat{v}, \hat{w}, \hat{p})=(u(z), v(z), w(z), p(z)) e^{i(\alpha r+\beta \operatorname{Re\theta }-\omega t)}
$$

Here the radial wavenumber $\alpha=\alpha_{r}+i \alpha_{i} \in \mathbb{C}$ and the azimuthal wavenumber and frequency $\beta, \omega \in \mathbb{R}$. It is assumed that $\beta$ is $O(1)$ and the number of spiral vortices around the disk surface is given by $n=\beta R e$. Furthermore, the orientation angle of the vortices with respect to a circle centred on the axis of rotation is defined as $\epsilon=\tan ^{-1}\left(\beta / \alpha_{r}\right)$. We set the frequency $\omega=0$ as we are interested in stationary vortices rotating with the rough disk.

The linearised perturbation equations are expressed in the primitive variables of equation (3) as

$$
\begin{gathered}
-\frac{1}{R e} \hat{u}^{\prime \prime}+\frac{R o}{R e} W \hat{u}^{\prime}+\left(i \alpha U+i \beta V+\frac{\alpha^{2}}{R e}+\frac{\beta^{2}}{R e}+\frac{R o}{R e} U\right) \hat{u}-\left(\frac{2 R o}{R e} V+\frac{C o}{R e}\right) \hat{v}+U^{\prime} \hat{w}+i \alpha \hat{p}=0 \\
-\frac{1}{R e} \hat{v}^{\prime \prime}+\frac{R o}{R e} W \hat{v}^{\prime}+\left(i \alpha U+i \beta V+\frac{\alpha^{2}}{R e}+\frac{\beta^{2}}{R e}+\frac{R o}{R e} U\right) \hat{v}+\left(\frac{2 R o}{R e} V+\frac{C o}{R e}\right) \hat{u}+V^{\prime} \hat{w}+i \beta \hat{p}=0 \\
-\frac{1}{R} \hat{w}^{\prime \prime}+\frac{R o}{R e} W \hat{w}^{\prime}+\left(i \alpha U+i \beta V+\frac{\alpha^{2}}{R e}+\frac{\beta^{2}}{R e}+\frac{R o}{R e} W^{\prime}\right) \hat{w}+\hat{p}^{\prime}=0 \\
\left(i \alpha+\frac{R o}{R e}\right) \hat{u}+\frac{i \beta}{R e} \hat{v}+\hat{w}^{\prime}=0
\end{gathered}
$$

and are solved directly using a Galerkin projection in terms of Chebyshev polynomials in the wall-normal direction. Equations (4)-(7) are then solved to obtain solutions of the dispersion relation $D(\alpha, \beta ; R e,[a, \eta])=0$ with the aim of studying the occurrence of convective instabilities for various values of the roughness parameters. The use of the Chebyshev polynomials ensures a higher accuracy compared to standard finite-difference methods with a similar discretisation. An exponential map is adopted to map the Gauss-Lobatto grid points, used for the Chebyshev polynomials, into the physical space: 100 points are therefore distributed between the lower disk surface $z=0$ and the top of the domain, $z_{\max }=20$. The stability equations are written and solved in primitive variables at all the collocation points but the ones at the boundaries $\left(z=0\right.$ and $\left.z=z_{\max }\right)$, where the boundary conditions $u(0)=v(0)=w(0)=w^{\prime}(0)=0$ and $u\left(z_{\max }\right)=v\left(z_{\max }\right)=w\left(z_{\max }\right)=p\left(z_{\max }\right)=0$ are enforced. This approach has two distinct advantages over the shooting and vorticity methods traditionally used in the literature ${ }^{16 ; 20 ; 28}$. 
Firstly, transformed variables are not required, and secondly all eigenvalues can be obtained at the same time rather than by searching iteratively from some initial guess. Note that equations (4)-(7) are entirely consistent with Lingwood's ${ }^{16}$ transformed equations (11)-(17).

Our spectral code has been verified against existing results in the literature for the BEK system ${ }^{16 ; 28}$ with a smooth lower disk, and also those due to Cooper et al. for the rough rotating disk. Very close agreement was found for the critical values of the Type I mode while some minor discrepancies were found for the Type II results. These discrepancies are consistent with the spread of values found in the literature for the Type II mode in the von Kármán system and, we expect, can be attributed to apparent sensitivity of the Type II results to the solution methods used. For example, we note that Balakumar \& Malik ${ }^{29}$ attributed their discrepancy with Malik's ${ }^{30}$ earlier prediction of the Type II critical Reynolds number to the use of primitive variables rather then a transformed vorticity formulation. A comparison between our results and those of Cooper et al. can be seen by comparing their Table I with our Table 1.

\subsection{Results and discussion}

As expected from the related literature, two instability modes are found to dictate the convective instability properties of all flows within the BEK family with lower surface roughness. The Type I mode arises from the inflectional behaviour of mean-flow components and appears as the upper lobe in $R e-\alpha_{r}$ neutral curves. In contrast, the Type II mode arises from the streamline curvature and Coriolis effects and appears as a lower lobe. Sample neutral curves resulting from our analyses are presented in Figures 5 and 6 and critical Reynolds numbers for the onset of both modes are shown in Tables $1-3$. Results are presented here for $R o=-1,0,1$ only, but the analysis has also been performed for $R o \in[-1,1]$ with a consistent progression in behaviour found between these model parameter values.

Figures 5(a) and 5(b) show neutral curves in the $R e-\alpha_{r}$ plane for the two cases of anisotropic roughness in the von Kármán flow. Furthermore, Figure 6(a) shows the equivalent neutral curve for isotropic surface roughness. We note that these are consistent with those of Cooper et al. and are included here for completeness only. We find that, in this case, each type of roughness acts to stabilise the Type I mode of instability, with particular sensitivity to azimuthally-anisotropic and isotropic roughness. In contrast, the Type II mode is stabilised by azimuthally-anisotropic and isotropic roughness, to the extent that the lower lobe is eliminated for even modest levels 


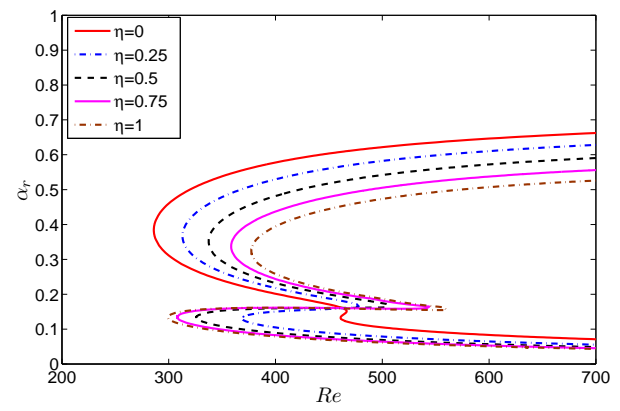

(a) von Kármán, $R o=-1$

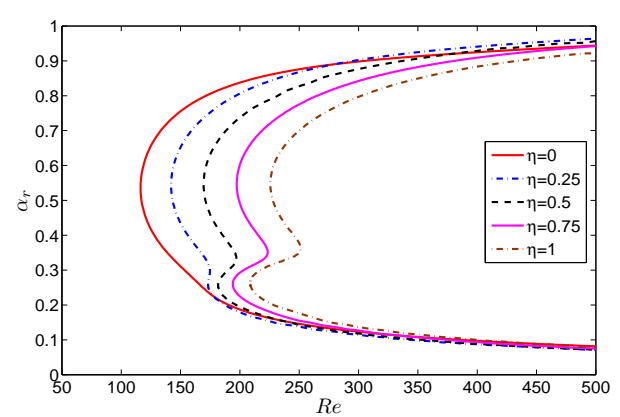

(c) Ekman, $R o=0$

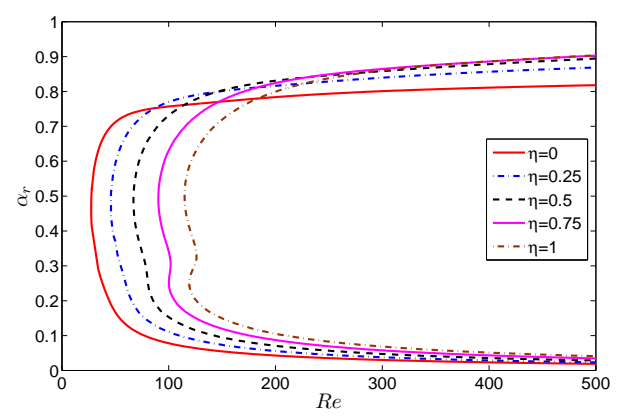

(e) Bödewadt, $R o=1$

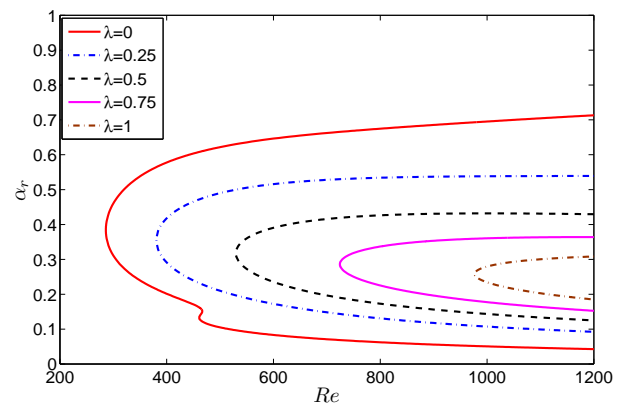

(b) von Kármán, $R o=-1$

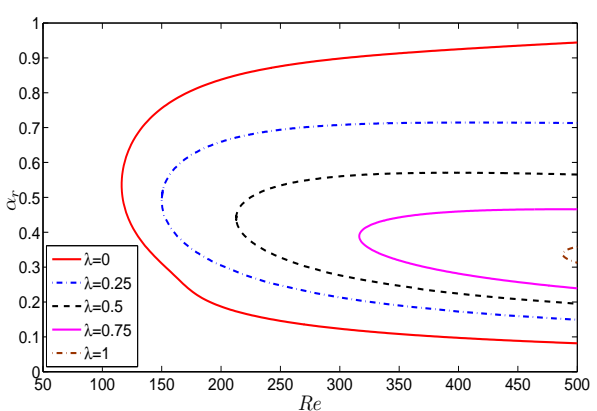

(d) Ekman, $R o=0$

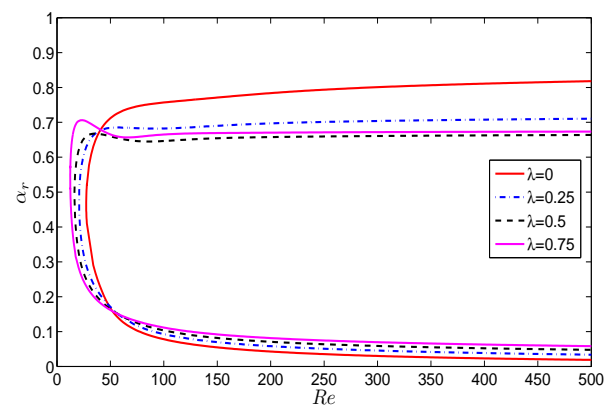

(f) Bödewadt, $R o=1$

Figure 5: Neutral curves for the BEK family over rough disks: (a)-(c)-(e) radially-anisotropic roughness (concentric grooves); (b)-(d)-(f) azimuthally-anisotropic roughness (radial grooves). 


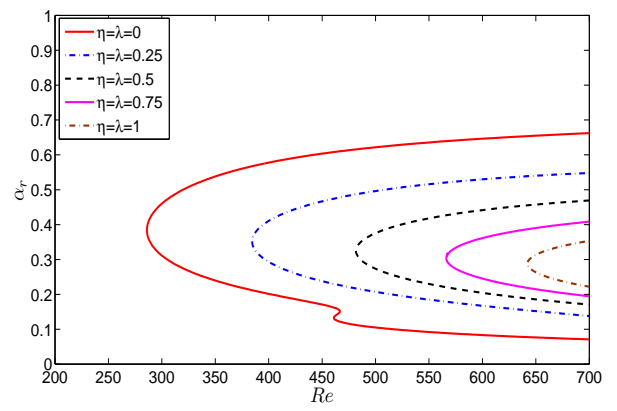

(a) von Kármán, $R o=-1$

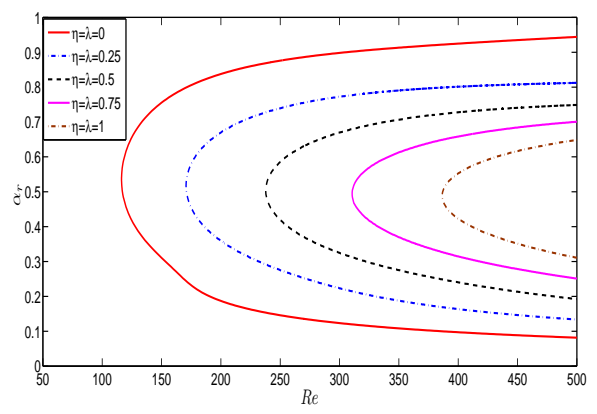

(b) Ekman, $R o=0$

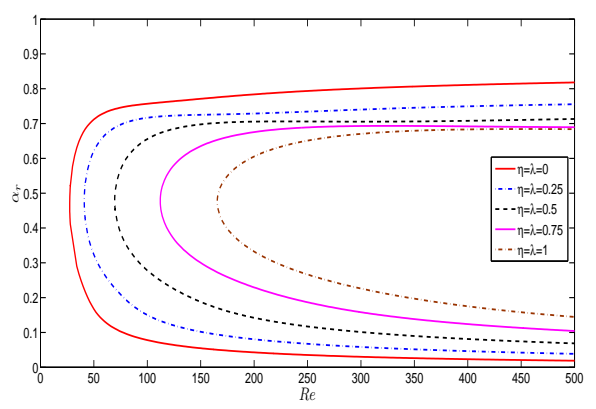

(c) Bödewadt, $R o=1$

Figure 6: Neutral curves for the BEK family over rough disks: isotropic roughness 


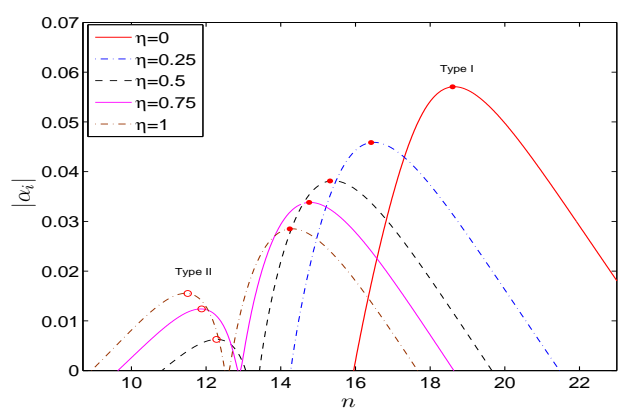

(a) Radially-anisotropic roughness

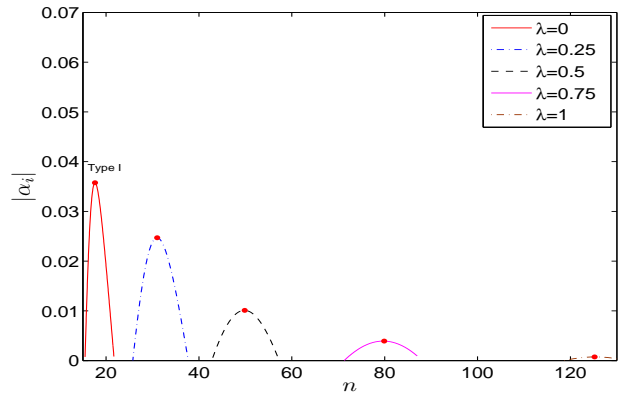

(b) Azimuthally-anisotropic roughness

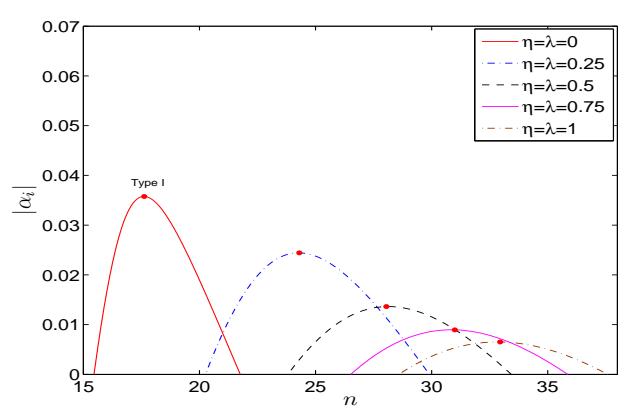

(c) Isotropic roughness

Figure 7: Growth rates of instability modes within the Ekman layer $(R o=1)$ as a function of vortex number $n$ at $R e=R e_{\text {critical }}+25$. The red dots indicate the most rapidly growing mode.

of roughness, but significantly destabilised by the radially-anisotropic roughness. A detailed analysis of these curves has been given by Cooper et al. ${ }^{4}$

Figures 5(c), 5(d) and 6(b) demonstrate that the response of the Ekman layer to surface roughness is similar to that of the von Kármán flow. That is, all roughness types lead to a stabilisation of the Type I mode with particular sensitivity to azimuthally-anisotropic and isotropic roughnesses. We again see that the Type II mode is destabilised by radially-anisotropic roughness. The effect is however less pronounced than for the von Kármán flow owing to the apparent insensitivity of the Ekman layer to the Type II mode of instability

Figures 5(e), 5(f) and 6(c) show some interesting behaviour for the Bödewadt flow. Isotropic roughness is again seen to be universally stabilising to both modes. Similarly, radially-anisotropic roughness is stabilising to the Type I mode but acts to promote the Type II mode. This general behaviour has been confirmed for all $R o \in[-1,1]$. However, in contrast to both the von Kármán and Ekman cases, the Bödewadt layer is found to be relatively insensitive to azimuthally-anisotropic roughness and the response is in fact mildly destabilising. Although 
not shown here, further investigation of the results for $R o \in[0,1]$ shows that the stabilising effect of azimuthally-anisotropic roughness is reversed at $R o=0.7$. That is, azimuthally-anisotropic roughness is stabilising for $R o \in[-1,0.7]$, with the reduced sensitivity with increased $R o$ until it leads to a destabilising effect over $R o \in(0.7,1]$.

A secondary interpretation of the neutral curves arises from consideration of the width of the unstable region in $\alpha_{r}$. Irrespective of the effect on the critical Reynolds number, all types of roughness are seen to narrow the region of instability, which is a stabilising effect in some sense. The width of the von Kármán and Ekman neutral curves are seen to be particularly sensitive to both isotropic and azimuthally-anisotropic roughness. We also note that the asymptotic behaviour of the lower branch of each neutral curve is apparently insensitive to radially-anisotropic roughness as $R e \rightarrow \infty$, but this is not the case for azimuthally-anisotropic or isotropic roughness.

Predicted critical values of the number of vortices $n$ and their angle of orientation $\epsilon$ are shown in Tables 1-3. These represent experimentally observable quantities, although unfortunately we are unaware of published experimental results for comparisons to be made with. The data demonstrates that radially-anisotropic roughness acts to reduce the number of vortices for both the von Kármán and Ekman cases. This is found to be true for all $R o \in[-1,0]$ with the sensitivity decreasing as Ro is increased. The results for the Bödewadt layer show the opposite behaviour; as radially-anisotropic roughness acts to increase the number of vortices. Further investigation shows that this change in behaviour occurs at $R o=0.7$. Similarly, the effect of azimuthally-anisotropic roughness is seen to be reversed at $R o=0.7$ with an increase in the predicted number of vortices for $R o \in[-1,0.7]$ and a decrease for $R o \in(0.7,1]$. Isotropic roughness is however seen to increase the number of vortices for all $R o$ with the apparent sensitivity increasing with $R o$. Similar results can be seen for the predictions of the orientation angle.

Complementary results arise from consideration of the effects of roughness on the growth rates, $\left|\alpha_{i}\right|$, of each instability mode. Figure 7 shows growth rates of the modes within the Ekman layer at $R e=R e_{\text {critical }}+25$, namely at a fixed distance from the neutral curve, for each level of roughness. Figure 7 (a) clearly shows the stabilising effect of radially-anisotropic roughness on the Type I mode and the destabilising effect on the Type II mode. Furthermore, the stabilising effect of both azimuthally-anisotropic and isotropic roughness can be seen in 
Table 1: Critical values of observable parameters at the onset of instability of both modes for the von Kármán flow. Type I and (Type II). The most dangerous mode is indicated as bold text in terms of critical Reynolds number.

\begin{tabular}{llll}
\multicolumn{5}{c}{ Radially-anisotropic roughness (concentric grooves) } \\
\hline Parameter & $R e$ & $n$ & $\epsilon$ \\
\hline$\eta=0$ & $\mathbf{2 8 6 . 0 5}(460.92)$ & $\mathbf{2 2 . 2 0}(21.28)$ & $\mathbf{1 1 . 4 0}(19.28)$ \\
$\eta=0.25$ & $\mathbf{3 1 2 . 9 1}(368.56)$ & $\mathbf{1 8 . 3 5}(13.27)$ & $\mathbf{9 . 0 6}(15.42)$ \\
$\eta=0.5$ & $337.42(\mathbf{3 2 5 . 8 0})$ & $\mathbf{1 6 . 0 2}(9.94)$ & $(7.66)(\mathbf{1 2 . 8 7})$ \\
$\eta=0.75$ & $358.48(\mathbf{3 0 7 . 7 9})$ & $\mathbf{1 4 . 4 0}(8.16)$ & $(6.77)(\mathbf{1 1 . 2 2})$ \\
$\eta=1$ & $377.36(\mathbf{2 9 9 . 2 4})$ & $\mathbf{1 3 . 1 6}(7.05)$ & $(6.11)(\mathbf{1 0 . 0 0})$ \\
& Azimuthally-anisotropic roughness (radial grooves) \\
\hline Parameter & Re & $n$ & $\epsilon$ \\
\hline$\lambda=0$ & $\mathbf{2 8 6 . 0 5}(460.92)$ & $\mathbf{2 2 . 2 0}(21.28)$ & $\mathbf{1 1 . 4 0}(19.28)$ \\
$\lambda=0.25$ & $\mathbf{3 8 0 . 6 8}(-)$ & $\mathbf{3 7 . 2 1}(-)$ & $\mathbf{1 5 . 4 1}(-)$ \\
$\lambda=0.5$ & $\mathbf{5 2 9 . 5 4}(-)$ & $\mathbf{6 0 . 1 2}(-)$ & $\mathbf{1 9 . 7 2}(-)$ \\
$\lambda=0.75$ & $\mathbf{7 2 4 . 3 6}(-)$ & $\mathbf{8 9 . 3 9}(-)$ & $\mathbf{2 3 . 4 6}(-)$ \\
$\lambda=1$ & $\mathbf{9 7 6 . 8 5}(-)$ & $\mathbf{1 3 0 . 3 9}(-)$ & $\mathbf{2 7 . 3 1}(-)$ \\
& \multicolumn{1}{c}{ Isotropic roughness } & $\epsilon$ \\
\hline Parameter & $R e$ & $n$ & $\mathbf{1 1 . 4 0}(19.28)$ \\
\hline$\eta=\lambda=0$ & $\mathbf{2 8 6 . 0 5}(460.92)$ & $\mathbf{2 2 . 2 0}(21.28)$ & $\mathbf{1 1 . 3 5}(-)$ \\
$\eta=\lambda=0.25$ & $\mathbf{3 8 4 . 5 3}(-)$ & $\mathbf{2 7 . 0 4}(-)$ & $\mathbf{1 0 . 5 4}(-)$ \\
$\eta=\lambda=0.5$ & $\mathbf{4 8 1 . 6 4}(-)$ & $\mathbf{2 9 . 1 3}(-)$ & $\mathbf{9 . 8 2}(-)$ \\
$\eta=\lambda=0.75$ & $\mathbf{5 6 6 . 2 4}(-)$ & $\mathbf{2 9 . 9 0}(-)$ & $\mathbf{9 . 0 5}(-)$ \\
$\eta=\lambda=1$ & $\mathbf{6 4 2 . 1 6}(-)$ & $\mathbf{2 9 . 5 7}(-)$ & \\
\hline \hline
\end{tabular}

Figures 7(b) \& (c), respectively. Such presentations of the growth rates can also be used to determine the most rapidly growing mode that is most likely to be observed in experiments. Figure $7(\mathrm{a})$ shows that the maximum growth rates shift to lower values of vortex number $n$ for both instability modes, indicating a reduction in the number of vortices over the disk with increasing levels of radially-anisotropic roughness. In contrast, despite the clear stabilising effect of the azimuthally-anisotropic and isotropic roughnesses, Figures 7(b) \& (c) show that the maximum growth rates shift to higher values of $n$, corresponding to an increase in the number of vortices under these roughness types. These conclusions are consistent with the behaviour of the critical parameters reported in Table 2.

Note that a detailed discussion of the growth rates for the von Kármán case has been given previously by Copper et al. and are not repeated here. Furthermore, the very early onset of absolute instability in the Bödewadt flow (as predicted by Lingwood \& Garrett) means that equivalent plots of the convective growth rates are not possible to produce. 
Table 2: Critical values of observable parameters at the onset of instability of both modes for the Ekman flow. Type I and (Type II). The most dangerous mode is indicated as bold text in terms of critical Reynolds number.

Radially-anisotropic roughness (concentric grooves)

\begin{tabular}{llllll}
\hline Parameter & $R e$ & \multicolumn{3}{l}{$n$} & $\epsilon$ \\
\hline$\eta=0$ & $116.26(-)$ & 16.04 & $(-)$ & 14.33 & $(-)$ \\
$\eta=0.25$ & $\mathbf{1 4 2 . 0 4}(173.17)$ & $\mathbf{1 4 . 6 8}(13.52)$ & $\mathbf{1 0 . 3 1}(16.61)$ \\
$\eta=0.5$ & $\mathbf{1 6 9 . 3 5}(181.65)$ & $\mathbf{1 4 . 0 2}(11.44)$ & $\mathbf{8 . 6 0}(14.89)$ \\
$\eta=0.75$ & $197.33(\mathbf{1 9 4 . 0 1})$ & $13.63(\mathbf{1 0 . 3 4})$ & $7.45 \quad(\mathbf{1 1 . 4 6})$ \\
$\eta=1$ & $225.67(\mathbf{2 0 8 . 6 5})$ & $13.38(\mathbf{9 . 6 4})$ & $5.73 \mathbf{( 9 . 7 4 )}$
\end{tabular}

Azimuthally-anisotropic roughness (radial grooves)

\begin{tabular}{|c|c|c|c|}
\hline Parameter & $R e$ & $n$ & $\epsilon$ \\
\hline$\lambda=0$ & $116.26(-)$ & $16.04(-)$ & $14.33(-)$ \\
\hline$\lambda=0.25$ & $149.99(-)$ & $26.60(-)$ & $19.48(-)$ \\
\hline$\lambda=0.5$ & $212.70(-)$ & $44.30(-)$ & $25.21(-)$ \\
\hline$\lambda=0.75$ & $316.38(-)$ & $73.55(-)$ & $30.37(-)$ \\
\hline$\lambda=1$ & $487.69(-)$ & $122.18(-)$ & $36.67(-)$ \\
\hline \multicolumn{4}{|c|}{ Isotropic roughness } \\
\hline Parameter & $R e$ & $n$ & $\epsilon$ \\
\hline$\eta=\lambda=0$ & $116.26(-)$ & $16.04(-)$ & $14.33(-)$ \\
\hline$\eta=\lambda=0.25$ & $170.66(-)$ & $21.45(-)$ & $13.75(-)$ \\
\hline$\eta=\lambda=0.5$ & $237.79(-)$ & $25.55(-)$ & $12.03(-)$ \\
\hline$\eta=\lambda=0.75$ & $310.63(-)$ & $28.65(-)$ & $10.89(-)$ \\
\hline$\eta=\lambda=1$ & $386.49(-)$ & $30.85(-)$ & $9.17(-)$ \\
\hline
\end{tabular}


Table 3: Critical values of observable parameters at the onset of instability of both modes for the Bödewadt flow. Type I and (Type II). The most dangerous mode is indicated as bold text in terms of critical Reynolds number.

\begin{tabular}{llll}
\multicolumn{4}{c}{ Radially-anisotropic roughness (concentric grooves) } \\
\hline Parameter & $R e$ & $n$ & $\epsilon$ \\
\hline$\eta=0$ & $\mathbf{2 7 . 3 8}$ & $\mathbf{3 . 1 2}(-)$ & $\mathbf{1 4 . 3 2}(-)$ \\
$\eta=0.25$ & $\mathbf{4 5 . 9 7}$ & $\mathbf{4 . 4 3}(-)$ & $\mathbf{1 2 . 0 3}(-)$ \\
$\eta=0.5$ & $\mathbf{6 7 . 0 0}$ & $\mathbf{5 . 5 5}(-)$ & $\mathbf{8 . 6 0}(-)$ \\
$\eta=0.75$ & $\mathbf{9 0 . 2 5}(100.70)$ & $\mathbf{6 . 4 1}(6.23)$ & $\mathbf{8 . 5 9}(12.61)$ \\
$\eta=1$ & $\mathbf{1 1 4 . 8 5}(119.21)$ & $\mathbf{7 . 0 7}(6.20)$ & $\mathbf{6 . 8 8}(11.46)$
\end{tabular}

Azimuthally-anisotropic roughness (radial grooves)

\begin{tabular}{|c|c|c|c|}
\hline Parameter & $R e$ & $n$ & $\epsilon$ \\
\hline$\lambda=0$ & $27.38(-)$ & $3.12(-)$ & $14.32(-)$ \\
\hline$\lambda=0.25$ & $20.82(-)$ & $2.53(-)$ & $14.90(-)$ \\
\hline$\lambda=0.5$ & $16.40(-)$ & $1.99(-)$ & $13.75(-)$ \\
\hline$\lambda=0.75$ & $12.41(-)$ & $1.45(-)$ & $12.03(-)$ \\
\hline \multicolumn{4}{|c|}{ Isotropic roughness } \\
\hline Parameter & $R e$ & $n$ & $\epsilon$ \\
\hline$\eta=\lambda=0$ & $27.38(-)$ & $3.12(-)$ & $14.32(-)$ \\
\hline$\eta=\lambda=0.25$ & $41.02(-)$ & $4.55(-)$ & $12.60(-)$ \\
\hline$\eta=\lambda=0.5$ & $69.50(-)$ & $7.22(-)$ & $12.03(-)$ \\
\hline$\eta=\lambda=0.75$ & $112.15(-)$ & $10.45(-)$ & $10.88(-)$ \\
\hline$\eta=\lambda=1$ & $165.52(-)$ & 13.64(-) & $9.74(-)$ \\
\hline
\end{tabular}




\section{Conclusion}

The study was designed to investigate the effects of anisotropic and isotropic surface roughnesses on the convective instability of stationary disturbances in the BEK family of flows. The partial-slip approach of Miklavčič \& Wang $^{26}$ was used without modification to model the steady boundary-layer flows within each flow configuration (as parameterised by Rossby number, Ro). The resulting mean-flow profiles are qualitatively consistent with those in the literature for the smooth cases at all Ro. Our study has revealed that radially-anisotropic (concentric grooves) and isotropic roughness acts to dampen the oscillatory behaviour of the steady flows up through the boundary layer and also reduces the magnitude of the wall jet. Azimuthally-anisotropic roughness (radial grooves) is however found to have the opposite effect, and this can be attributed to the flows (which naturally move in the radial direction) being channelled and accelerated by the radial grooves.

We performed linear stability analyses and revealed that radially-anisotropic roughness results in the stabilisation of Type I mode of instability at all Ro. In contrast, this class of roughness acts to promote the Type II mode at all Ro, to the extent that that mode becomes the dangerous mode for sufficiently negative $R o$ and sufficiently large roughness parameter. Azimuthally-anisotropic roughness was found to have a strong stabilising effect on both instability modes for $R o \in[-1,0.7]$, although it was marginally destabilising for $R o \in(0.7,1]$. In contrast, isotropic roughness was shown to be universally stabilising to both mode types for all flows.

The conclusions arising from the study are clear. Surface roughness has been found to be a potentially effective passive flow-control methodology within engineering flows of the rotorstator type modelled by the BEK system and also other cross-flow dominant flows. Furthermore, the apparent sensitivity to the precise design of the surface roughness is a rich area for further study.

A number of parallels between this current study and that of Lingwood \& Garrett ${ }^{28}$ can be drawn. The most significant is that surface roughness is an alternative stabilising mechanism to mass flux that is likely to be technologically easier to implement and more robust. Furthermore, as with mass flux, the precise response of the physical mechanisms that lead to transition in the boundary layer is sensitive to the Rossby number of the system. 
Rough surfaces are of course expected to select stationary disturbances within rotating boundary-layer flows such as was considered here. For this reason, we have not considered travelling convective instability modes or absolute instability.

This research used the ALICE High Performance Computing Facility at the University of Leicester. SJG is supported by a Senior Research Fellowship of the Royal Academy of Engineering, funded by the Leverhulme Trust. BA wishes to acknowledge financial support from the Ministry of National Education, Republic of Turkey.

\section{Bibliography}

[1] L. Sirovich and S. Karlsson. Turbulent drag reduction by passive mechanisms. Nature, 388(6644):753-755, 1997.

[2] P. Carpenter. The right sort of roughness. Nature, 388(6644):713-714, 1997.

[3] K. Choi. Fluid dynamics: The rough with the smooth. Nature, 440(7085):754-754, 2006.

[4] A.J. Cooper, J.H. Harris, S.J. Garrett, M. Özkan, and P.J. Thomas. The effect of anisotropic and isotropic roughness on the convective stability of the rotating disk boundary layer. Physics of Fluids (1994-present), 27(1):014107, 2015.

[5] T. von Kármán. Über laminare und turbulente reibung. Z. Angew. Math. Mech., 1921.

[6] U.T. Bödewadt. Die drehströmung über festem grunde. ZAMM-Journal of Applied Mathematics and Mechanics/Zeitschrift für Angewandte Mathematik und Mechanik, 20(5):241$253,1940$.

[7] V. W. Ekman. On the influence of the Earth's rotation on ocean currents. Ark. Mat. Astron. Fys., 2:1-53, 1905.

[8] Ö. Savas. Stability of Bödewadt flow. Journal of Fluid Mechanics, 183:77-94, 1987.

[9] J.M. Lopez. Flow between a stationary and a rotating disk shrouded by a co-rotating cylinder. Physics of Fluids (1994-present), 8(10):2605-2613, 1996. 
[10] R.J. Lingwood and P.H. Alfredsson. Experimental study of the stability of the Bödewadt layer. Springer, 2000.

[11] L. Schouveiler, P. Le Gal, and M.P. Chauve. Instabilities of the flow between a rotating and a stationary disk. Journal of Fluid Mechanics, 443:329-350, 2001.

[12] A. Cros, E. Floriani, P. Le Gal, and R. Lima. Transition to turbulence of the Batchelor flow in a rotor/stator device. European Journal of Mechanics-B/Fluids, 24(4):409-424, 2005.

[13] N.A. Culverhouse. The hydrodynamic stability of crossflow vortices in the Bödewadt boundary layer. $\mathrm{PhD}$ thesis, University of Birmingham, 2009.

[14] P.R. Tatro and E.L. Mollo-Christensen. Experiments on Ekman layer instability. Journal of Fluid Mechanics, 28(03):531-543, 1967.

[15] A.J. Faller. An experimental study of the instability of the laminar Ekman boundary layer. Journal of Fluid Mechanics, 15(04):560-576, 1963.

[16] R.J. Lingwood. Absolute instability of the ekman layer and related rotating flows. Journal of Fluid Mechanics, 331:405-428, 1997.

[17] B.I. Fedorov, G.Z. Plavnik, I.V. Prokhorov, and L.G. Zhukhovitskii. Transitional flow conditions on a rotating disk. Journal of Engineering Physics and Thermophysics, 31(6):1448$1453,1976$.

[18] T.C. Corke and K.F. Knasiak. Stationary travelling cross-flow mode interactions on a rotating disk. Journal of Fluid Mechanics, 355:285-315, 1998.

[19] R.J. Lingwood and P.H. Alfredsson. Instabilities of the von Kármán boundary layer. Applied Mechanics Reviews, 67(3):030803, 2015.

[20] A.J. Cooper and P.W. Carpenter. The stability of rotating-disc boundary-layer flow over a compliant wall. part 1. type I and II instabilities. Journal of Fluid Mechanics, 350:231-259, 1997.

[21] A.J. Colley, P.J. Thomas, P.W. Carpenter, and A.J. Cooper. An experimental study of boundary-layer transition over a rotating, compliant disk. Physics of Fluids (1994-present), 11(11):3340-3352, 1999. 
[22] S. Jarre, P. Le Gal, and M.P. Chauve. Experimental study of rotating disk flow instability. ii. forced flow. Physics of Fluids (1994-present), 8(11):2985-2994, 1996.

[23] S. Imayama, P. H. Alfredsson, and R.J. Lingwood. On the laminar-turbulent transition of the rotating-disk flow: the role of absolute instability. Journal of Fluid Mechanics, 745:132-163, 2014.

[24] T. Watanabe, H.M. Warui, and N. Fujisawa. Effect of distributed roughness on laminarturbulent transition in the boundary layer over a rotating cone. Experiments in fluids, 14(5):390-392, 1993.

[25] S.J. Garrett, Z. Hussain, and S.O. Stephen. The cross-flow instability of the boundary layer on a rotating cone. Journal of Fluid Mechanics, 622:209-232, 2009.

[26] M. Miklavčič and C.Y. Wang. The flow due to a rough rotating disk. Zeitschrift für angewandte Mathematik und Physik ZAMP, 55(2):235-246, 2004.

[27] M. S. Yoon, J. M. Hyun, and Jun Sang P. Flow and heat transfer over a rotating disk with surface roughness. International journal of heat and fluid flow, 28(2):262-267, 2007.

[28] R.J. Lingwood and S.J. Garrett. The effects of surface mass flux on the instability of the bek system of rotating boundary-layer flows. European Journal of Mechanics-B/Fluids, $30(3): 299-310,2011$.

[29] P. Balakumar and M.R. Malik. Traveling disturbances in rotating-disk flow. Theoretical and Computational Fluid Dynamics, 2(3):125-137, 1990.

[30] M. R. Malik. The neutral curve for stationary disturbances in rotating-disk flow. Journal of Fluid Mechanics, 164:275-287, 1986. 